\title{
Fördert Mittagsschlaf das Gedächtnis?
}

Ein Mittagsschlaf soll nicht nur den Körper entspannen, sondern auch dem Gehirn die Aufnahme neuer Informationen erleichtern. Angeblich wird das Kurzzeitgedächtnis besser.

— Forscher der Berkeley University of California haben die Effekte des Mittagsschlafs in einer Studie überprüft. 39 junge Erwachsene wurden in zwei Gruppen aufgeteilt und sollten sich dort einem Lerntest unterziehen. Dazu mussten die Studienteilnehmer Fakten lernen und sich möglichst viele merken.

In dem zur Mittagszeit abgehaltenen Test erreichten beide Gruppen annähernd gleiche Leistungen. Am gleichen Tag durfte die eine Hälfte $a b$ 14 Uhr 90 Minuten schlafen, die andere blieb wach. Um $18 \mathrm{Uhr}$ folgte der nächste Test. Diesmal erreichte die Gruppe, die geschlafen hatte, ein besseres Ergebnis. Sie schnitt sogar besser $a b$ als im vorausgegangenen Test.

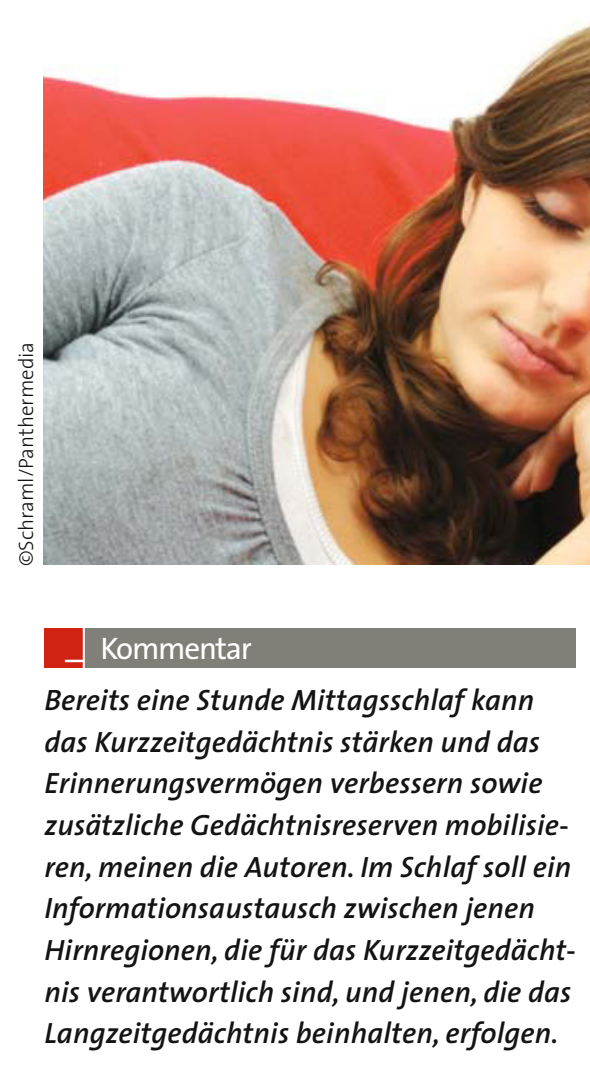

Frisch erworbene Informationen werden im Schlaf verarbeitet und langfristig gespeichert.

K. MALBERG =
Nur ein Nickerchen oder Informationsverarbeitung?

\section{Angststörung: Lavendelöl so stark wie ein Benzodiazepin?}

Dem Lavendel wird eine beruhigende und schlaffördernde Wirkung nachgesagt. Jetzt wurde untersucht, ob ein standardisiertes, orales Lavendelpräparat bei generalisierten Angstzuständen hilft.

- Deutsche Forscher randomisierten 77 Patienten mit generalisierten Angstzuständen in zwei Gruppen. In dieser Double-Dummy-Studie erhielt die Experimentalgruppe sechs Wochen lang Kapseln mit 80 mg Lavendelöl $\left(\right.$ Silexan $\left.^{\circledR}\right)$, während die Kontrollgrupe über den gleichen Zeitraum mit 0,5 mg Lorazepam behandelt wurde.

Die Ergebnisse zeigen, dass sich der Wert auf der Hamilton Anxiety Rating
Scale, dem primären Endpunkt, in beiden Gruppen ähnlich positiv veränderte. Die sekundären Studienendpunkte, z.B. Schlafqualität, zeigten analoge Entwicklungen. Ernste Nebenwirkungen waren in keiner Gruppe zu beklagen. Die Autoren schließen aus diesen Daten, dass Silexan ${ }^{\circledR}$ bei dieser Indikation wirksam ist.

\section{Kommentar}

Lavendelöl wird schon lange von Aromatherapeuten gegen Nervosität, Angst oder Schlafstörungen eingesetzt. Unklar war allerdings, ob bei dieser Applikationsform das Öl oder die sanfte Massage das Wirkprinzip beinhalten. Die vorliegende Studie scheint die erste Untersuchung zu sein, bei der das Öl oral verabreicht wurde. Die Ergebnisse sind ermutigend und implizieren, dass Lavendel ebenso wirksam wie ein Benzodiazepin ist. Da diese Untersuchung jedoch die erste ihrer Art ist und zudem für eine Äquivalenzstudie eine recht kleine Fallzahl aufweist, sollten wir mit weitreichenden Schlussfolgerungen eher vorsichtig sein. Fazit: Ermutigend, aber mehr Daten wären schön.

E. ERNST =

\footnotetext{
- H. Woelk, S. Schläfke

A multi-center, double-blind, randomised study of the lavender oil preparation Silexan in comparison to Lorazepam for generalized anxiety disorder. Phytomedicine 17 (2010) 94-99
} 